\title{
Creation and Validation of a Structured Training Program to Train ICU Nurses in Free Flap Monitoring
}

\author{
Samir S. Khariwala, MD, MS ${ }^{1}$ Sandra Alexander, RN, MBA, MSN² Bevan Yueh, MD, MPH${ }^{1}$ \\ ${ }^{1}$ Department of Otolaryngology-Head and Neck Surgery, University of \\ Minnesota, Minneapolis, Minnesota \\ 2 Intensive Care Unit, University of Minnesota Medical Center, \\ Minneapolis, Minnesota \\ Address for correspondence Samir S. Khariwala, MD, MS, MMC 396, \\ 420 Delaware St, Minneapolis, MN 55455 \\ (e-mail: Khari001@umn.edu).
}

J Reconstr Microsurg Open 2016;1:8-11.

\begin{abstract}
Background Monitoring is an important part of the postoperative care of free flap patients. Because the burden of flap examinations often falls to the house staff, residents must make special trips to the hospital for flap examinations, which increases duty hours. We developed a training program designed to train intensive care unit (ICU) nurse to perform significant portion of free flap monitoring.

Methods First, ICU charge nurses received a slide presentation and completed an online module to consolidate the didactic training. Second, the nurses were given a written reference manual describing the basic concepts behind free tissue transfer and normal flap physiology. Finally, "on-the-job" training of the nurses was done by residents/house staff. Flap-related outcomes were analyzed.

Results The entire group of 24 ICU nurses targeted for this education were successfully trained and transitioned to nurse-led monitoring. For the first 50 patients monitored by ICU nurses following this educational effort, we had no adverse events and no signs of flap compromise were missed by ICU charge nurses. In one instance, an ICU nurse required a resident to personally examine a flap. There were no partial or total flap losses.

Conclusions Training of ICU nurses in free flap monitoring can be accomplished

Keywords

- free flap

- monitoring

- flap salvage

- ICU nurse through the following principles: (1) leadership and support by the microsurgeons involved; (2) faculty-led didactic and web-based training modules; (3) significant involvement of the otolaryngology house staff; and (4) consistent and experienced ICU nurses in collaboration with nursing leadership. This is the first description of a successfully validated curriculum.
\end{abstract}

For patients with complex defects in the head and neck resulting from malignancy, or extirpation of it, free tissue transfer is often the reconstructive method of choice.

This manuscript was presented as an oral paper on September 22, 2014, at the American Academy of Otolaryngology-Head and Neck Surgery Annual Meeting (Orlando, Florida, September 21-24, 2014).
Success rates for free tissue transfer are routinely cited to be at or above 90 to $95 \%{ }^{1}$ The high rate of success is the result of a gradual increase in the cumulative experience of microsurgeons, improved instrumentation and advancing technology in microscopes, and intensive early monitoring of free flap viability. In recent years, the performance of free tissue reconstruction in otolaryngology departments has received

May 8, 2015

accepted after revision

August 23, 2015

published online

January 21, 2016
DOI http://dx.doi.org/

10.1055/s-0035-1570534. ISSN 2377-0813.
Copyright $\odot 2016$ by Thieme Medical

Publishers, Inc., 333 Seventh Avenue,

New York, NY 10001, USA.

Tel: +1(212) 584-4662.
License terms

(요 (1) $\Theta \circledast$ 
gradually increased such that there are 1.6 microsurgeons per department and free tissue surgeons make up an average of $12 \%$ of the departmental faculty. ${ }^{2}$ As a result, free tissue reconstruction experience for otolaryngology trainees has gone from unusual to very common over a 15to 20-year period.

While techniques and protocols for flap monitoring vary widely, data suggest that early periodic examinations of free tissue transfers allow for developing problems to be identified early enough such that flap salvage is possible. ${ }^{3}$ Given the fact that vascular insufficiency can develop quite suddenly in the early postoperative period, flap examinations should occur frequently in the early postoperative period, to offer the best possible chance for flap salvage in the event of a microvascular obstruction.

At teaching institutions, the burden of free tissue monitoring is most commonly borne by the house staff. With the advent of duty hour restrictions for trainees, the need for multiple flap examinations overnight can make compliance difficult. We sought to identify an alternate means of providing excellent postoperative observation of flap health, while minimizing resident burden. Many centers have developed a "step-down unit" for flap monitoring, but for a variety of reasons, this was not possible at the University of Minnesota Medical Center. Because patients receiving free flaps require intensive monitoring, our protocol is to keep these patients in the intensive care unit (ICU) for the first 48 hours after surgery, with the nurse-to-patient ratio of $1: 2 .^{4}$ We sought to develop a physician-led curriculum to train a core team of ICU nurses to perform these duties. This article describes the material and strategies utilized to implement the new approach as well as initial experience following implementation.

\section{Methods}

Training of nurses consisted of three steps. First, we identified a core group of high-quality, accountable nurses with low turnover. At our institution, the charge nurses in the ICU represented a suitable group that met our criteria. We then worked with the hospital administration and nursing leadership to gain approval for our initiative. Once approved, we instituted the nursing training program.

The initial step in training was a didactic session consisting of a PowerPoint presentation by a faculty microsurgeon (S. S. K.) to this group of ICU charge nurses. The presentation covered basic topics relating to microvascular free tissue transfer, including basic flap physiology, the nature microvascular anastomoses, common donor sites, and common indications for transfer. We also briefly discussed postoperative monitoring as well as normal and abnormal postoperative bedside examination findings. Lastly, we showed a short video of a "flap check" being performed by one of the faculty members.

The second step in the training regimen was the development of a "reference manual," which was uploaded to a web site accessible to all nurses. The manual is a seven-page document that included background information on free

\section{Summary Checklist}

\section{Please check the following during each flap check}

\author{
Flap color
}

Doppler

$\square$ Needle poke (if ordered)

Temperature to touch

Turgor (Is the flap soft to the touch?)

\section{Neckinspection}

\section{$\square$ Head position}

Fig. 1 Summary checklist for free flap examinations.

flaps, monitoring protocols and day-by-day descriptions of normal appearance, signs of concern, and a summary checklist for each flap check (see - Supplementary Table 1 available in the online version of the article; - Fig. 1). The day-by-day description was intended to bring attention to some finer points in free flap physiology during the early postoperative period. For example, nurses were taught to understand that a flap that appeared somewhat pale the first 24 hours was usually normal and that flaps would often slowly become pinker over the coming days as the microvasculature dilated. Importantly, we sought to teach the nurses that examination of a flap is a skill that requires consideration of multiple data points which include color, arterial/venous Doppler, turgor, warmth, needle poke, etc., to determine an accurate overall picture. They were also taught that, in general, a single data point (i.e., arterial Doppler) was rarely sufficient to determine the true status of the flap.

The last component of the nurse training was performed with the assistance of the house staff and consisted of "onthe-job" training. To accomplish this, the house staff called the charge nurse on duty to the bedside at the time of each flap check during the day or night (to increase the chances for all charge nurses to gain experience) so that the check could be performed in the presence of the nurse. The resident discussed all the pertinent findings with the charge nurse and helped them to develop an overall picture of the flap status (see - Supplementary Table 2 available in the online version of the article). Each charge nurse was required to document 10 instances of flap checks performed with residents. Each nurse had a checklist that documented the time and date of each check. After completion of the 10th supervised check, the ICU nurses were considered "certified."

After such time that all ICU charge nurses had completed their training with house staff and were deemed "certified," the transition to nurse-led flap checks was initiated, starting 
on postoperative day (POD) 1. Because the checks are so critical on the first evening, we continued resident-only checks on the first night following surgery (POD 0). However, starting on PODs 1 to 4, flap checks between 10 PM and 6 AM were performed by the ICU charge nurse using a "virtual flap check." The virtual flap check consisted of the ICU nurse performing the examination while on the telephone with the resident on-call so that the findings could be discussed in real time. If both the nurse and the on-call resident felt comfortable with the findings as reported by the nurse, no further action was required. If, on the other hand, the ICU nurse expressed concern or was unsure of the appearance of the free flap, the resident was called to the bedside for an inperson examination. Although a description of the educational framework used is the primary focus of this effort, we collected data on the first 50 free tissue transfers monitored on the new protocol. Therefore, there were no specific enrollment criteria for this secondary study aim. The study was approved by the University of Minnesota Institutional Review Board (study \# 1403M49022). We documented missed findings by the nurse, flap returns to the operating room, flap loss, neck hematoma, events requiring house staff flap examination after nurse exam, and number of resident flap exams replaced by "virtual flap checks" performed by ICU nurses.

\section{Results}

A group of 24 ICU nurses was enrolled in the planned training curriculum. All 24 nurses completed this successfully including the 10 checks performed in conjunction with house staff. In our first 50 cases of ICU nurse-assisted flap checks, there were no missed findings of flap compromise. There was only one instance in which the nurse was uncomfortable with the appearance of the flap, and the resident on-call was summoned to the bedside. Subsequent examination performed by the resident revealed that the flap was healthy.

More importantly, no flap losses were experienced during this period. One patient was taken back to the operating room due to loss of the implantable Doppler signal and poor blood return on needle poke. Exploration revealed that the implantable Doppler had become dislodged but the anastomoses were patent and the flap healed without issues.

We estimated the time saved for the on-call house staff as a result of the new flap monitoring protocol. Since the residents performed the free flap checks on the evening of surgery, this program had no impact on the first night (POD 0). Our program requires two checks on POD 1 (10 PM, 2 AM) and then one check on POD 2 and POD 3 (midnight), so the ICU nurse-assisted flap checks saved the residents four trips to the hospital per patient. Our residents live at variable distances from the hospital and sometimes come in from other hospitals, so it was not possible to compile the data with exact time figures. However, each flap examination was conservatively estimated to require 45 minutes for the resident to drive to the hospital, park, walk to the bedside, perform the examination, document the examination, walk back to the parking area, and drive home. At 45 minutes per examination, $180(4 \times 45)$ minutes, or 3 hours, were saved per patient. Over the course of the 50 patients studied here, this amounts to 150 hours of time saved.

\section{Discussion}

Our study demonstrates that a curriculum and training program designed to train ICU nurses in free tissue monitoring can be successfully developed and implemented. Once successfully implemented, the use of ICU nurse-led free tissue monitoring has the potential to assist in adherence to house staff duty hour restrictions. ICU nurse-assisted flap checks are feasible and, in our initial experience, did not result in adverse outcomes. We anticipate that this trend will continue as the nurses become more experienced and comfortable with free flap monitoring.

Evidence suggests that trainees in Otolaryngology-Head and Neck Surgery are commonly exposed to free tissue reconstruction in training programs ${ }^{2}$ and that rates of flap loss are exceedingly low in experienced centers. A postoperative flap monitoring is an important factor in the high rate of success. This has led to an increase in free flap volumes and the requisite postoperative monitoring. With the advent of duty hour limitations for house staff, the workload associated with postoperative free flap monitoring has become difficult to manage in some training programs. This is especially true for programs in which residents take overnight call from home and must travel to the hospital for each bedside flap examination.

At our institution, overnight free flap examinations required the residents to make single or multiple trips into the hospital for free flap examination only. This required evening/ night trips to the hospital specifically for bedside free flap examinations such that compliance with duty hour restrictions became a challenge. As such, we identified a need to limit the burden placed on house staff relating to free tissue monitoring. Many institutions have utilized "step-down" units which usually function as semiclosed units where nurses working there become very familiar with head and neck patients having undergone specific procedures. In the case of free tissue transfer, the nurses can become very familiar with free tissue and, over time, their experience reaches a point where they can successfully monitor a free flap without direct surveillance. While Khan et $\mathrm{al}^{5}$ described the use of a cartoon and teaching sessions to familiarize nurses with free flaps, our study is the first of its kind to define a structured program that successfully trains general ICU nurses to monitor flaps without physicians in the room.

Jackson et al studied two groups of patients receiving free flaps, one monitored primarily by nurses and one monitored primarily by physicians. ${ }^{6}$ In each group, complications, length of stay, reoperations, and flap viability were studied. All of these variables were similar in both groups, so the authors suggested that there were no negative effects in having free flaps monitored by nursing. Given that the use of ICUs is common in the postoperative management of patients receiving free flaps, ${ }^{7}$ we feel that 
the training protocols presented in this study are easily translatable to many institutions where these types of procedures are performed. In many cases, either a stepdown unit or ICU would be suitable for this type of free flap monitoring protocol.

It should be noted that advancements in technology have enabled physicians to become more comfortable with the possibility of nonphysicians monitoring free tissue transfers. Indeed, there are now multiple tools available for free flap monitoring that are used to varying degrees on an institution-to-institution basis. Some of the techniques currently used include: implantable Doppler, microendoscopy, externalization of a flap segment, laser Doppler flow meter, tissue oxygenation wonders, and surface temperature monitors, in addition to many others. ${ }^{8}{ }^{8}$ In our institution, we currently use an implantable Doppler as an adjunctive tool. While we counsel the house staff and nurses that the presence or absence of implantable Doppler signal is not, in and of itself, a definitive determinant of flaps status, it does act as another piece of objective data which can be used by nurses and physicians when determining the overall status of the free flap. In the future, technology including tissue oximetry sensors may act as an adjunct or even replacement for current protocols for clinical examination. ${ }^{10}$ Tissue oximetry in particular has been studied in non-head and neck reconstruction but only to a lesser degree in head and neck cases. ${ }^{11}$ Thus, additional data are needed to support widespread implementation of this technology in head and neck reconstruction.

One potential weakness of this study is that, although we did not identify any negative outcomes after we transitioned to flap checks performed by the ICU nurses, our numbers are relatively small given the low rate of flap complications. We acknowledge that the study of complications in free tissue transfer is inherently difficult because rates of complications are quite low, and therefore it is possible that reporting of additional cases may at some point identify a missed finding by a nurse. However, we believe that this possibility could also occur with resident-only examinations.

In summary, we have developed a successful strategy and protocol for training ICU nurses to perform free flap monitoring in the early postoperative period. Our protocol utilizes a clear stepwise approach with significant physician involvement to ensure that nurses reach a high level of comfort and expertise prior to performing examinations on their own. In addition, we have instituted the use of the "virtual flap check" which keeps the house staff involved and available for immediate assistance when necessary. Moving forward, we hope to begin adding the POD 0 examinations to the current schedule of ICU nurse-led free flap monitoring in the near future. We feel that this approach is safe and ensures positive patient outcomes while allowing training programs to more easily adhere to house staff duty hour restrictions and improve resident satisfaction.

\section{References}

1 Suh JD, Sercarz JA, Abemayor E, et al. Analysis of outcome and complications in 400 cases of microvascular head and neck reconstruction. Arch Otolaryngol Head Neck Surg 2004;130(8): 962-966

2 Spiegel JH, Polat JK. Microvascular flap reconstruction by otolaryngologists: prevalence, postoperative care, and monitoring techniques. Laryngoscope 2007;117(3):485-490

3 Chubb D, Rozen WM, Whitaker IS, Acosta R, Grinsell D, Ashton MW. The efficacy of clinical assessment in the postoperative monitoring of free flaps: a review of 1140 consecutive cases. Plast Reconstr Surg 2010;125(4):1157-1166

4 Cornejo A, Ivatury S, Crane CN, Myers JG, Wang HT. Analysis of free flap complications and utilization of intensive care unit monitoring. J Reconstr Microsurg 2013;29(7):473-479

5 Khan MA, Mohan A, Ahmed W, Rayatt S. Nursing monitoring and management of free and pedicled flaps-outcomes of teaching sessions on flap care. Plast Surg Nurs 2010;30(4):213-216, quiz 217-218

6 Jackson RS, Walker RJ, Varvares MA, Odell MJ. Postoperative monitoring in free tissue transfer patients: effective use of nursing and resident staff. Otolaryngol Head Neck Surg 2009;141(5): 621-625

7 Marsh M, Elliott S, Anand R, Brennan PA. Early postoperative care for free flap head \& neck reconstructive surgery-a national survey of practice. Br J Oral Maxillofac Surg 2009;47(3):182-185

8 Luu Q, Farwell DG. Advances in free flap monitoring: have we gone too far? Curr Opin Otolaryngol Head Neck Surg 2009;17(4): 267-269

9 Abdel-Galil K, Mitchell D. Postoperative monitoring of microsurgical free tissue transfers for head and neck reconstruction: a systematic review of current techniques-part I. Non-invasive techniques. Br J Oral Maxillofac Surg 2009;47(5):351-355

10 Lohman RF, Langevin CJ, Bozkurt M, Kundu N, Djohan R. A prospective analysis of free flap monitoring techniques: physical examination, external Doppler, implantable Doppler, and tissue oximetry. J Reconstr Microsurg 2013;29(1):51-56

11 Lin SJ, Nguyen MD, Chen C, et al. Tissue oximetry monitoring in microsurgical breast reconstruction decreases flap loss and improves rate of flap salvage. Plast Reconstr Surg 2011;127(3): 1080-1085 